\title{
MRAC and MPC Controllers for Load Application System of the Accelerated Testing Equipment of Pavements
}

\author{
Oscar Javier Reyes Ortiz ${ }^{\mathrm{a}, 1}$, Juan Sebastian Useche Castelblanco ${ }^{\mathrm{a}, 2}$, German Leandro Vargas Fonseca ${ }^{\mathrm{a}, 3}$ \\ ${ }^{a}$ Engineering School, Universidad Militar Nueva Granada, Carrera 11 101-80, Bogota, 110111, Colombia \\ E-mail: ${ }^{\prime}$ oscar.reyes@unimilitar.edu.co, ${ }^{2}$ u7700125@unimilitar.edu.co, ${ }^{3}$ german.vargas@unimilitar.edu.co
}

\begin{abstract}
For the study of pavement structures, different methodologies and devices have been used and those have been modified and modernized. The development of these routes directly impacts the social and economic development of the different regions. Through the roads, the interconnection between different points is allowed, and the resources that need to be invested for its construction are high. Trends show real-scale studies to determine real operating parameters that allow improving design processes. For this reason, test devices have been developed that simulating real operating conditions, but these machines require robust and efficient control. Adaptive and predictive controls are the most used in industrial processes, where it is necessary to reduce performance and operation costs-obtaining smooth transitions in the control signal, especially when techniques are used with followup to reference models. This document shows the design of the MRAC (Adaptive Reference Control Model) and MPC (Predictive Control Model) controller applied to a hydraulic loading system for real-scale pavement test equipment. The mathematical development of the plant and the controllers is presented, along with its implementation, simulation, and analysis. The main objective of this work is to verify the effectiveness of these controllers for this type of real scale system since due to the number of variables that affect these devices and the complexity of the study material.
\end{abstract}

Keywords — MRAC; MPC; laguerre; lyapunov; pavements.

\section{INTRODUCTION}

The asphalt and hydraulic mixtures are the elements used throughout the world for the construction of roads that connect different sites and regions [1]. This infrastructure has a direct impact on the social and economic development of a country due to the investments involved and the integration of the territory. Therefore, the importance of their study in terms of its mechanical and dynamic behavior [2]. For a detailed study in this area in the last decades, real-scale tests have been generated. These tests show the behavior of the road with real conditions of loads and operating, allowing optimize natural resources and reduce costs [3]. Some problems in the control of this equipment are the large amounts of energy consumed, the external disturbances, the acquisition systems, and the study variables (loads, deformations, temperature, stresses, and accelerations) [4].

Currently, adaptive and predictive controls are the most used in industrial processes where it is necessary to reduce costs of performance and operation. Especially the controllers that are focused on working with reference models like the MRAC or the MPC [5], [6]. Model Reference Adaptive Control (MRAC), establishes an external model to be followed by the plant [7]. The MRAC works ensuring that the error converges to 0 , using an adaptation algorithm that does not require total mathematical knowledge of the plant [8], [9]. One algorithm solution is the implementation of the Lyapunov stability law [10]. Model Predictive Control (MPC) is the basic prediction system but has been most successful in the industry [11]. Its optimization process ensures stability and convergence to the desired equilibrium point. It is necessary to know the mathematical model of the plant in its entirety in order to predict it [12], [13]. Some related papers with these controllers are The development of a MPC for a hydraulic car system where the versatility of the controller against external disturbances is evident [14]. The control of a hydraulic valve using a MPC [15] shows that this type of controllers has good behavior in non-linear and multivariable systems. The development of an MRAC is presented by a previous study [16], where a precision control in the position of actuators type SMA is implemented. This type of controllers has a high performance in trajectory tracking. The authors work with this type of adaptive systems for the control of an electro-pneumatic actuator where it is demonstrated that this type of control helps to improve the speed of response of the system [17].

This paper presents the design of an MRAC and MPC for a loading application system for the accelerated testing 
equipment of pavements. This dispositive is developed by the Geotechnical research group of the Universidad Militar Nueva Granada, from which all the physical parameters for the model will be extracted. In the following sections, the mathematical model of the hydraulic system is obtained, the controllers are developed; the MRAC uses the stability of Lyapunov and the MPC with discrete Laguerre functions. Finally, the signals of the plant response, control, and error are obtained and analyzed to conclude about the efficiency of this type of controllers.

\section{MATERIALS AND METHOD}

\section{A. Load Application System Model}

The load application system is observed in Fig. 1. The system works through a hydraulic circuit that moves two cylinders that produce a force between 0.5 and 8 tons. The load is transmitted to the pavement by two truck-type tires.

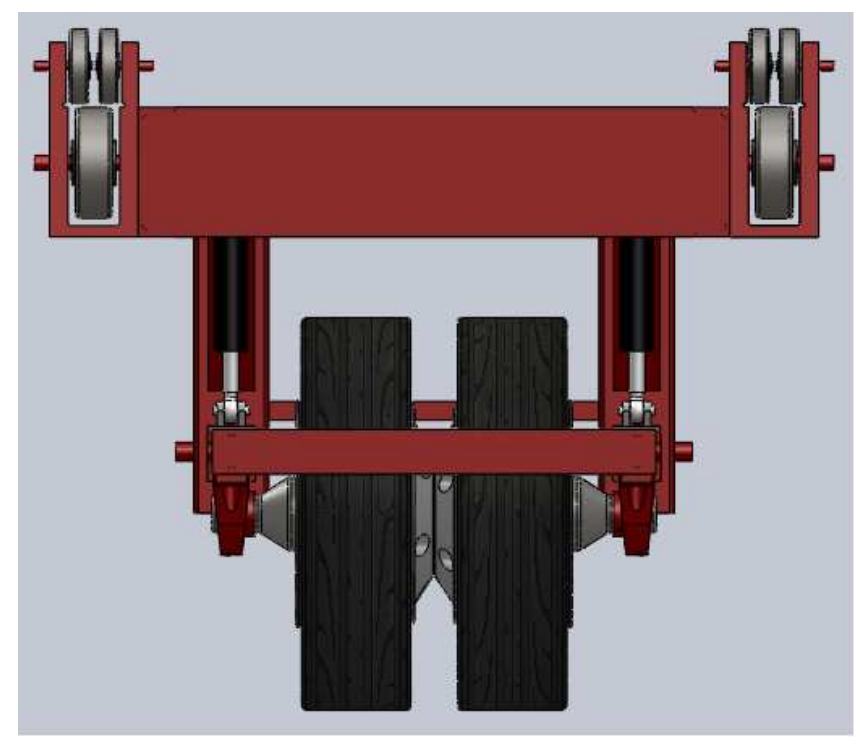

Fig. 1. Load Application System

The hydraulic system of Fig. 2 is responsible for generating the force on the tires. The circuit is composed of 4 parts: The first is the hydraulic unit that contains an AC motor and a variable vane pump with a constant flow of 0.36 $\mathrm{L} / \mathrm{s}$. The second is the proportional pressure relief valve, which, according to a voltage reference, maintains a constant pressure in the system. The third part is a hydraulic accumulator, which reduces the peaks of system flow and an electro-valve for manual control of the cylinders. The fourth part is composed of the pressure sensor and the hydraulic cylinder. The force generated is equal to the pressure of the system multiplied by the area of the plunger of the actuator [18].

In accordance with the operating principle of the constant flow vane pump, not taking into account the Coulomb friction and assuming the oil as an incomprehensible fluid [19], the system model is performed. Performing the summation of forces that occur in the hydraulic cylinder, Eq. 1 is obtained.

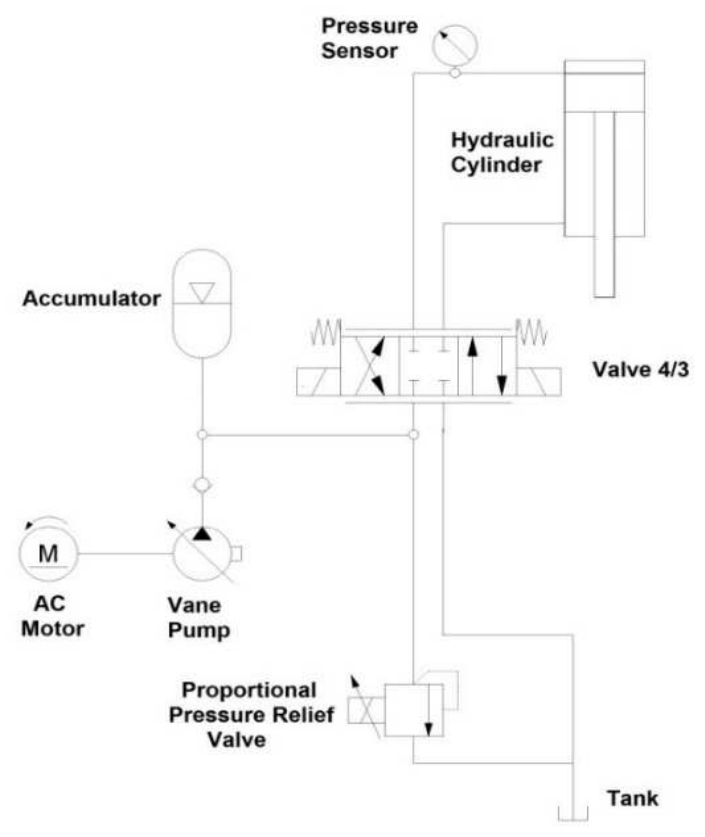

Fig. 2. Hydraulic System

$$
p A_{2}-p_{c} A_{1}=M \ddot{x}+B_{c} \dot{x}+k_{e} x
$$

Where, $p$ and $p_{c}$ are the pressures of the cylinder inlet and outlet chamber. The inlet pressure is the internal pressure of the system. $A_{1}$ and $A_{2}$ are the small and large crosssectional areas of the cylinder. $x$ is the displacement of the cylinder. $M$ is the mass of the rod-piston assembly. $\mathrm{B}_{\mathrm{c}}$ is the viscous resistance coefficient. $k_{\mathrm{e}}$ is the rigidity of the cylinder coupling. $p_{c}$ is the output pressure to the tank, this pressure is considered low in comparison to the working pressure $p$ as to get to affect it [15].

The output flow of the relief valve must respond to the displacement of the spool $(y)$. Performing the forces analysis acting on the spool, it is obtained Eq.2.

$$
\left(p_{0}-p\right) \cdot A=m \ddot{y}+B_{s} \dot{y}+k_{s} \cdot y
$$

$p_{0}$ is the pressure reference. A is the cross-sectional area of the spool. $\mathrm{m}$ is the spool mass. $B_{S}$ is the viscosity coefficient and $k_{s}$ represents the stiffness of the spool spring. As shown in Eq. 3, the pressure reference of the valve is proportional to the input voltage with a gain $\left(k_{v}\right)$. This is because the valve has an internal regulation system.

$$
p_{0}=k_{v} \cdot v
$$

Eq. 4 expresses the direct flow to the cylinder.

$$
Q_{E}=K_{Q} x-K_{l} p-\frac{V_{b}}{\beta_{e}} \dot{p}
$$

$K_{Q}$ is the flow constant; $K_{l}$ is the leakage constant; $\mathrm{V}_{\mathrm{b}}$ is the volume of the entry chamber y $\beta_{\mathrm{e}}$ is the oil bulk module. The input flow $\left(Q_{E}\right)$ is equal to $Q_{B}-Q_{S}$ where the first term is the constant flow of the pump, and the second is the output flow through the proportional relief valve. The mathematical function of the valve is expressed in Eq. 5.

$$
Q_{S}=k_{q} y+k_{c 1} p
$$

$k_{q}$ is the constant that relates the displacement of the spool 
with the valve opening hole. $k_{c 1}$ is the pressure discharge coefficient. Organizing the system into equations of state:

$$
\begin{gathered}
\dot{x}=v_{x} \\
\dot{v}_{x}=-\frac{k_{e}}{M} x-\frac{B_{c}}{M} v_{x}+\frac{A_{2}}{M} p \\
\dot{y}=v_{y} \\
\dot{v_{y}}=-\frac{k_{s}}{m} y-\frac{B_{s}}{m} v_{y}-\frac{A}{m} p+-\frac{A k_{v}}{m} v \\
\dot{p}=\frac{K_{Q} \beta_{e}}{V_{b}} x+\frac{\beta_{e} k_{q}}{V_{b}} y-\frac{\beta_{e}\left(K_{l}+k_{c 1}\right)}{V_{b}} p-\frac{\beta_{e} Q_{B}}{V_{b}}
\end{gathered}
$$

Table I shows the values of the system parameters.

TABLE I

SYSTEM PARAMETERS

\begin{tabular}{|c|l|l|}
\hline Parameter & \multicolumn{1}{|c|}{ Description } & \multicolumn{1}{c|}{ Value } \\
\hline$M$ & Rod-piston mass & $1.63 \mathrm{~kg}$ \\
\hline$m$ & Spool mass & $0.33 \mathrm{~kg}$ \\
\hline$K_{Q}$ & Flow constant & $3.72 \mathrm{e}-3 \mathrm{~m} 2$ \\
\hline$K_{l}$ & Leakage constant & $8.3 \mathrm{e} 3 \mathrm{~m} 3 / \mathrm{MPa}$ \\
\hline$V_{b}$ & $\begin{array}{l}\text { The volume of the entry } \\
\text { chamber }\end{array}$ & $2.13 \mathrm{e}-3 \mathrm{~m} 3$ \\
\hline$\beta_{e}$ & Fluid bulk module & $1.2 \mathrm{e} 3 \mathrm{MPa}$ \\
\hline$k_{e}$ & $\begin{array}{l}\text { The rigidity of the cylinder } \\
\text { coupling }\end{array}$ & $9.74 \mathrm{e} 2 \mathrm{~N} / \mathrm{m}$ \\
\hline$A_{2}$ & $\begin{array}{l}\text { The large cross-section area of } \\
\text { the piston }\end{array}$ & $1.56 \mathrm{e}-3 \mathrm{~m} 2$ \\
\hline$B_{c}$ & Viscous resistance coefficient & $0.45 \mathrm{~N} * \mathrm{~S} / \mathrm{m}$ \\
\hline$k_{q}$ & Spool - opening hole relation & $0.0697 \mathrm{~m} 2 / \mathrm{s}$ \\
\hline$k_{c 1}$ & $\begin{array}{l}\text { Coefficient of discharge by } \\
\text { pressure }\end{array}$ & $8.05 \mathrm{e}-9 \mathrm{~m} 3 / \mathrm{Pa}$ \\
\hline$A$ & Cross-section area spool & $3.12 \mathrm{e}-5 \mathrm{~m} 2$ \\
\hline$B_{S}$ & Spool viscosity coefficient & $0.38 \mathrm{~N} / \mathrm{m}$ \\
\hline$k_{S}$ & The rigidity of the spool spring & $3.7 \mathrm{e} 2 \mathrm{~N} / \mathrm{m}$ \\
\hline$k_{v}$ & Voltage-pressure expressed gain & $1.4 \mathrm{e} 6 \mathrm{~Pa} / \mathrm{V}$ \\
\hline & & \\
\hline
\end{tabular}

The response of the plant to a step input is illustrated in Fig. 3 . The graph shows a stable behavior with error in steadystate. Its settling time is around 12 seconds.

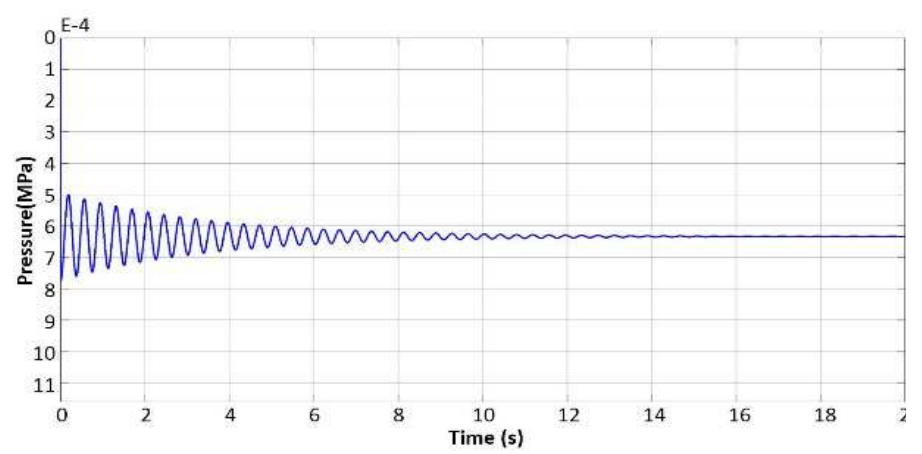

Fig. 3. Open-loop plant

\section{B. MRAC Using the Stability of Lyapunov}

The MRAC is performed based on the development of Lyapunov stability exhibited [20] y and its implementation for a control system [21]. The reference model and the process are in second-order differential equations, as shown in Eq. 7 and Eq. 8. The process is of a higher order, but an adaptation to this behavior is sought, and through the openloop response of the system is identified that it is possible.

$$
\begin{gathered}
\ddot{y_{m}}+a_{1 m} \dot{y_{m}}+a_{0 m} y_{m}=b_{m} r \\
\ddot{y}+a_{1} \dot{y}+a_{0} y=b u
\end{gathered}
$$

The Lyapunov control law is the difference between the input signal and the output of the plant, multiplied by the parameter $\theta$. The error is the subtraction between the process and the reference model. The above is shown in the Eq. 9 and Eq. 10.

$$
\begin{gathered}
u=\theta_{1} r+\theta_{2} y \\
e=y-y_{m}
\end{gathered}
$$

Deriving the error and replacing Eq. 7 and Eq. 8 as a function of the first derivatives, the following expression is obtained

$$
\dot{e}=\frac{\left(b u-\ddot{y}-a_{0} y\right)}{a_{1}}-\frac{\left(b_{m} r-\ddot{y_{m}}-a_{0 m} y_{m}\right)}{a_{1 m}}
$$

Replacing in the previous equation $y_{m}=e-y$ and $\ddot{y_{m}}=\ddot{e}-\ddot{y}$, the system is expanded as shown in the equation below

$$
\begin{aligned}
& \frac{1}{a_{1 m}} \ddot{e}+\dot{e}+\frac{a_{m 0}}{a_{1 m}} e \\
& =\frac{b \theta_{1} r}{a_{1}}-\frac{b_{m} r}{a_{1 m}}-\frac{b \theta_{2} y}{a_{1}}-\frac{a_{0} y}{a_{1}} \\
& +\frac{a_{0 m} y}{a_{1 m}}-\frac{\ddot{y}}{a_{1}}+\frac{\ddot{y}}{a_{1 m}}
\end{aligned}
$$

When the plant model is adapted to the reference system, constants are equal, in this way $a_{1}=a_{1 m}, a_{0}=a_{0 m}, b=$ $b_{m}$. Replacing these conditions in Eq. 12 and solving, it is obtained the differential Eq. 13 that expresses the behavior of the error in the adaptation.

$$
\dot{e}=-\frac{1}{a_{1 m}} \ddot{e}-\frac{a_{m 0}}{a_{1 m}} e+\frac{\left(b_{m} \theta_{1}-b_{m}\right)}{a_{1 m}} r-\frac{b_{m} \theta_{2}}{a_{1 m}} y
$$

The quadratic function of Eq. 14, it is introduced to determine the Lyapunov stability and calculate the parameters of adaptation.

$$
\begin{aligned}
V\left(e, \theta_{1}, \theta_{2}\right)= & \frac{1}{2}\left(a_{1 m} e^{2}+\frac{1}{b_{m} \gamma}\left(b_{m} \theta_{1}-b_{m}\right)^{2}\right. \\
& \left.+\frac{1}{b_{m} \gamma}\left(b_{m} \theta_{2}\right)^{2}\right)
\end{aligned}
$$

The derivative of the function is illustrated in Eq. 15. The system is stable when the derivative of the function is negative.

$$
\begin{gathered}
\frac{d V}{d t}=a_{1 m} e \frac{d e}{d t}+\frac{1}{\gamma}\left(b_{m} \theta_{1}-b_{m}\right) \frac{d \theta_{1}}{d t} \\
+\frac{1}{\gamma}\left(b_{m} \theta_{2}\right) \frac{d \theta_{2}}{d t}
\end{gathered}
$$

Replacing the derivative of the error of Eq. 13, it is defined the Eq. 16. 


$$
\begin{gathered}
\frac{d V}{d t}=a_{1 m} e\left[-\frac{1}{a_{1 m}}-\ddot{e}-\frac{a_{m 0}}{a_{1 m}} e+\frac{\left(b_{m} \theta_{1}-b_{m}\right)}{a_{1 m}} r\right. \\
\left.-\frac{b_{m} \theta_{2}}{a_{1 m}} y\right]+\frac{1}{\gamma}\left(b_{m} \theta_{1}-b_{m}\right) \frac{d \theta_{1}}{d t} \\
+\frac{1}{\gamma}\left(b_{m} \theta_{2}\right) \frac{d \theta_{2}}{d t}
\end{gathered}
$$

Factoring Eq. 17.

$$
\begin{aligned}
\frac{d V}{d t}=-a_{m} e^{2} & +\frac{1}{\gamma}\left(b_{m} \theta_{1}-b_{m}\right)\left(\frac{d \theta_{1}}{d t}+\gamma r e\right) \\
& +\frac{1}{\gamma}\left(b_{m} \theta_{2}\right)\left(\frac{d \theta_{2}}{d t}-\gamma y e\right)
\end{aligned}
$$

The derivative is negative if the parameter values are updated for that the expressions are equal to 0 . This is true, and the derivative will be equal to $-a_{m} e^{2}$ when the following equations are met.

$$
\begin{gathered}
\frac{d \theta_{1}}{d t}=-\gamma r e \\
\frac{d \theta_{2}}{d t}=\gamma y e
\end{gathered}
$$

Therefore, the value of the derivative is semi-defined, where error values and the parameters are limited. The value $\gamma$ can be varied to adjust the adaptation of the system. In this way, the method solves the Lyapunov stability problems presented using gradients. Applying the integral to the previous functions 18 and 19, the parameters for adaptation of the plant are as shown below

$$
\begin{gathered}
\theta_{1}=\frac{-\gamma_{1}}{b} \int r e d t+\theta_{1}(0) \\
\theta_{2}=\frac{\gamma_{2}}{b} \int y e d t+\theta_{2}(0)
\end{gathered}
$$

The reference model representing the desired behavior of the plant. For its design should consider the response time in open loop and the following equations.

$$
\begin{gathered}
M R=\frac{w_{n}{ }^{2}}{s^{2}+2 \rho w_{n} s+w_{n}{ }^{2}} \\
w_{n}=\frac{4.6}{\rho t_{s}}
\end{gathered}
$$

$\rho$ is the damping constant of the system, 4.6 represents the time constant, where the plant is within $1 \%$ error.

\section{MPC using Laguerre Functions}

The mathematical model of prediction by model is done based on the development of Laguerre functions discussed in [22], the process of minimizing the cost function [23] and obtaining the control matrix [24]. For system control by the state, space is implemented a MPC using Laguerre functions in discrete time. The general expression of vector control is given by Eq. 24. Each $\Delta U$ represents an independent vector prediction.

$$
\Delta U=[\Delta U(k i) \Delta U(k i+1) \ldots \Delta U(N c-1)]^{T}
$$

Where Nc represents the control horizon and the vector dimension in a sampling time $\mathrm{k}$, Laguerre functions are used to obtain approximate values of the vector $\Delta U$-rewriting the function as shown in Eq. 25.

$$
\Delta U=[\delta(i) \delta(i+1) \ldots \delta(N c-1)]^{T} \Delta U
$$

The elements of the vector $\Delta U$ can be expressed as a polynomial approximation of a discrete function. The functions are shown in Eq. 26.

$$
\begin{gathered}
\Gamma_{1}(z)=\frac{\sqrt{1-a^{2}}}{1-a z^{-1}} \\
\Gamma_{2}(z)=\frac{\sqrt{1-a^{2}}}{1-a z^{-1}} \frac{z^{-1}-a}{1-a z^{-1}} \\
\Gamma_{N c}(z)=\frac{\sqrt{1-a^{2}}}{1-a z^{-1}}\left(\frac{z^{-1}-a}{1-a z^{-1}}\right)^{N c-1}
\end{gathered}
$$

The value of " $a$ " determines the pole of the Laguerre network. It is used to ensure system stability. The parameter takes values in the interval of $0 \leq a_{i} \leq 1$, to observe the general behavior is set to 0.5 .

Applying the inverse $\mathrm{Z}$ transform as in Eq. 27, it is obtained the vector in discrete-time formed by $L_{i}(k)$ that contains the Laguerre functions from $l_{1}(k)$ to $l_{N}(k)$.

$$
l_{m}(k)=Z^{-1}\left\{\Gamma_{m}(Z)\right\}
$$

The operation of the networks in discrete-time satisfies the difference equation shown below:

$L(k+1)=A_{l} L(k)$

$A_{l}$ is a matrix of $(N x N)$ which groups the parameters of $a$ and $\beta=\left(1-a^{2}\right)$ as expressed in Eq. 29.

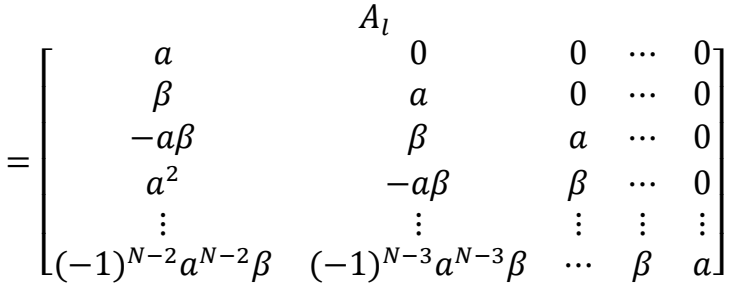

Eq. 30 establishes an initial condition of system operation to $L_{i}(k)$.

$$
L(0)^{T}=\sqrt{\beta}\left[\begin{array}{lll}
1 & -a & a^{2}-a^{3}(-1)^{N-1} a^{N-1}
\end{array}\right]
$$

The prediction of the future states and outputs of the plant using the Laguerre network is expressed in the following equations

$$
\begin{aligned}
& x(k i+m \mid k i)= A^{m} x(k i)+\sum_{i=0}^{m-1} A^{m-i-1} B L(i)^{T} n \\
& y(k i+m \mid k i)=C A^{m} x(k i) \\
&+\sum_{i=0}^{m-1} C A^{m-i-1} B L(i)^{T} n
\end{aligned}
$$

The vector $n$ is obtained from the response of the plant using the minimization of the cost function that is expressed in Eq. 33. The cost function is made using the prediction of the states within the prediction window $\mathrm{Np}$. 


$$
\begin{aligned}
J=n^{T}\left(\sum_{m=1}^{N p} \emptyset(m) Q \emptyset(m)^{T}+R_{L}\right) n \\
+2 n^{T}\left(\sum_{m=1}^{N p} \emptyset(m) Q A^{m}\right) x(k i) \\
+\sum_{m=1}^{N p} x(k i)^{T}\left(A^{T}\right)^{m} Q A^{m} x(k i)
\end{aligned}
$$

$Q=C^{T} C$ represents the output vector. $R_{L}$ is the matrix of the reference signals with a dimension of $(1 \mathrm{x} \mathrm{Np})$ as seen in Eq. 34. With Eq. 35 is obtained the matrix $\emptyset(m)$, this is the one that involves the model of the plant and the vector that contains the Laguerre functions.

$$
R_{L}=\left[\begin{array}{llll}
1 & 1 & \ldots & 1
\end{array}\right] r(k i)
$$

$=\sum_{j=0}^{m-1} A^{m-i-1}\left[B_{1} L_{1}(j)^{T} B_{2} L_{2}(j)^{T} B_{m} L_{m}(j)^{T}\right]$

By minimizing the cost function using its derivative, the vector of coefficients is expressed, as shown below.

$$
n=-\Omega^{-1} \Psi x(k i)
$$

$\Omega$ and $\Psi$ are the matrices of optimization of the inputs and the plant, respectively. Its obtain is expressed in Eq. 37 and Eq.38.

$$
\begin{gathered}
\Omega=\sum_{m=1}^{N p} \emptyset(m) Q \emptyset(m)^{T}+R_{L} \\
\Psi=\sum_{m=1}^{N p} \emptyset(m) Q A^{m}
\end{gathered}
$$

The control matrix is obtained by multiply the minimization and optimization matrices, with initial conditions of Laguerre functions, as illustrated in Eq. 39.

$$
\Delta U(k)=-K_{M P C}=-L(0)^{T} \Omega^{-1} \Psi
$$

With the control matrix, the closed-loop system is exposed below.

$$
x(k+1)=\left(A-B K_{M P C}\right) x(k)
$$

\section{RESULTS AND DISCUSSION}

Fig. 4 represents the two different control loops for the same system. The adaptive controller (MRAC) uses the error seen from the reference model and not from the setpoint. The predictive controller (MPC) uses the conventional error and requires the feedback of all system state variables. For the controls, the differential equations of the plant by blocks are implemented.

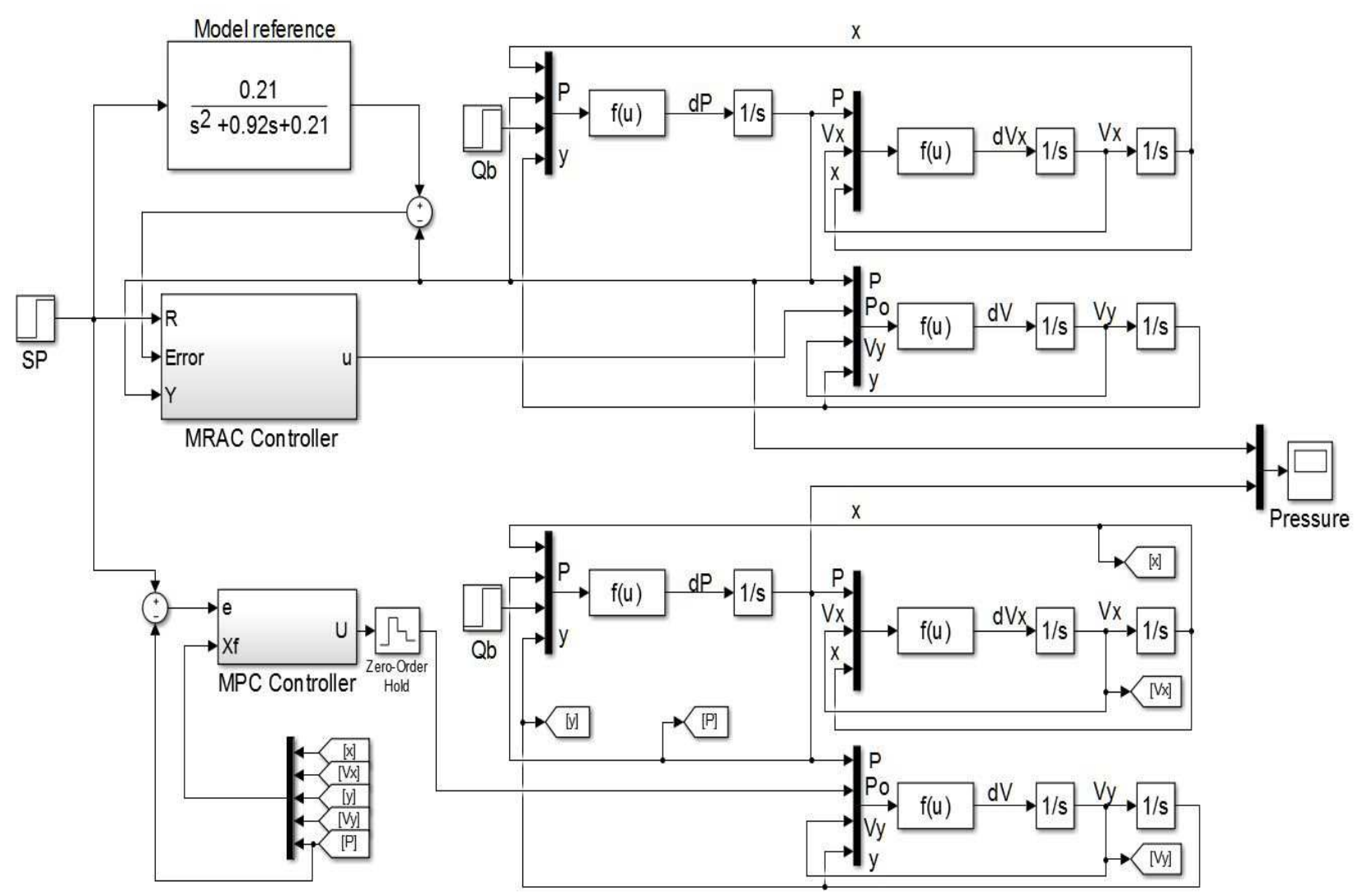

Fig. 4. MRAC and MPC control systems 
The values of the parameters used for the different controllers are shown in Table II.

TABLE II

CONTROL PARAMETERS

\begin{tabular}{|c|l|c|}
\hline \multicolumn{3}{|c|}{ MRAC controller } \\
\hline Parameter & \multicolumn{1}{|c|}{ Description } & Value \\
\hline$\gamma_{1 / b}, \gamma_{2 / b}$ & Adaptive adjustment & 5,1 \\
\hline $\mathrm{ts}$ & $\begin{array}{l}\text { The stabilization time of the } \\
\text { reference model }\end{array}$ & $20 \mathrm{seg}$ \\
\hline $\mathrm{p}$ & Damping of the reference model & 1 \\
\hline \multicolumn{3}{|c|}{ MPC controller } \\
\hline Parameter & \multicolumn{1}{|c|}{ Description } \\
\hline$N_{C}$ & $\begin{array}{l}\text { Control horizon, equal to the } \\
\text { system order }\end{array}$ & 5 \\
\hline$N_{P}$ & $\begin{array}{l}\text { Horizon prediction, equal to } 10 \\
\text { times the stabilization time of } \\
\text { the plant }\end{array}$ & 120 \\
\hline$a$ & Laguerre pole & 0.9 \\
\hline
\end{tabular}

Fig. 5 shows the response of the two controllers with a step input and in Fig. 6 the response to the input of a block. In addition to the graphs, the response of the plant with the PID controller currently implemented in the equipment is included.

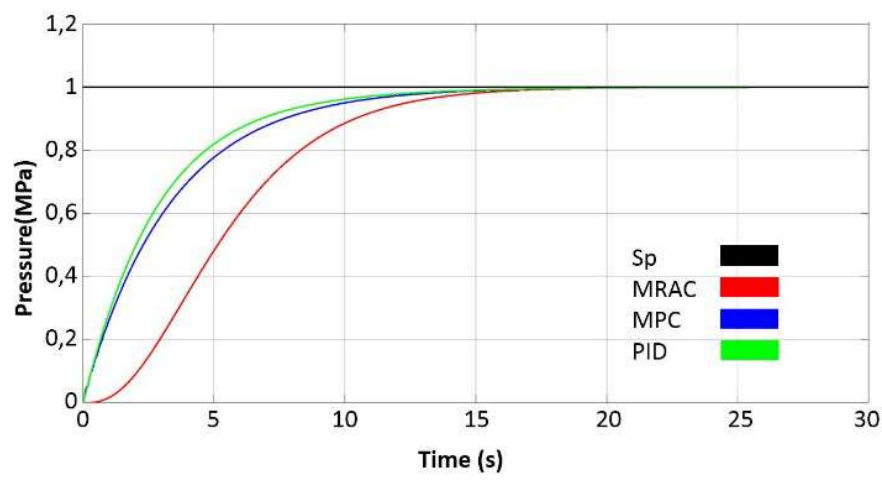

Fig. 5. Response to step input

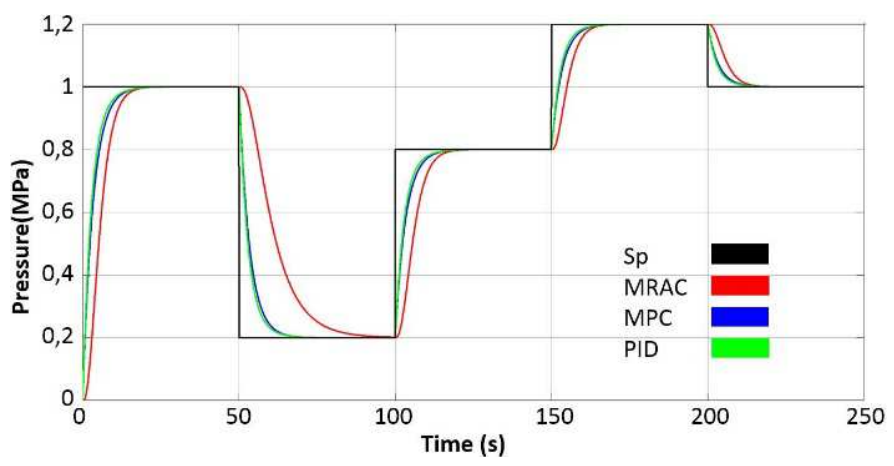

Fig. 6. Response to the input of a block

For the three cases, the same response is appreciated for positive and negative error values. The system responds critically damped in a stabilization time of 18 seconds. Fig. 7 and Fig. 8 show the control signals of the systems for the previous cases.

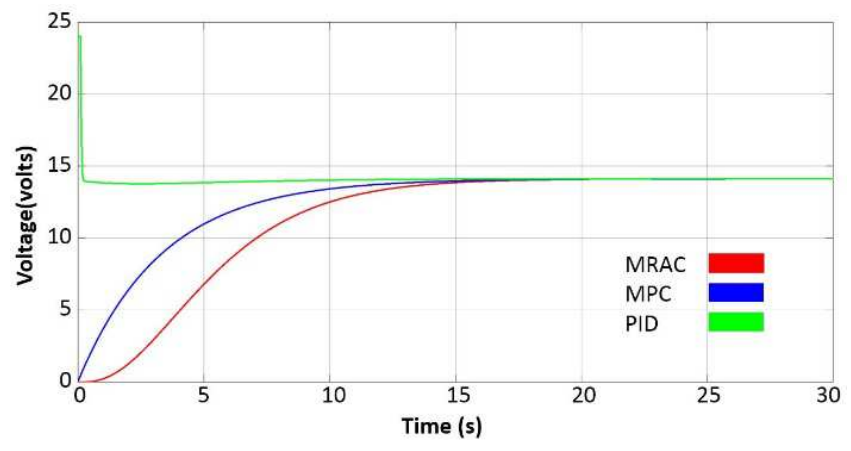

Fig. 7. The control signal to step input

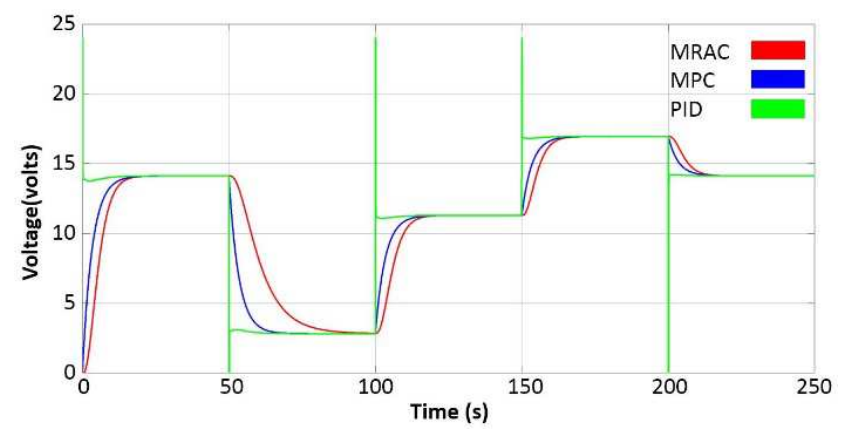

Fig. 8. The control signal to block input

The control signal is consistent with the response for a valve opening system for pressure regulation. In this case, the output values are lower than $24 \mathrm{DCV}$, which is the maximum control power of the valve. The figure also shows the difference in the systems control signal with a reference model and a traditional one. Fig. 9 shows the response of the plant to a disturbance in system pressure. This can be caused by the hydraulic pump, the change in the volume of the oil in the hydraulic actuator, or caused by the reaction between the pavement and the tires of the system, etc.

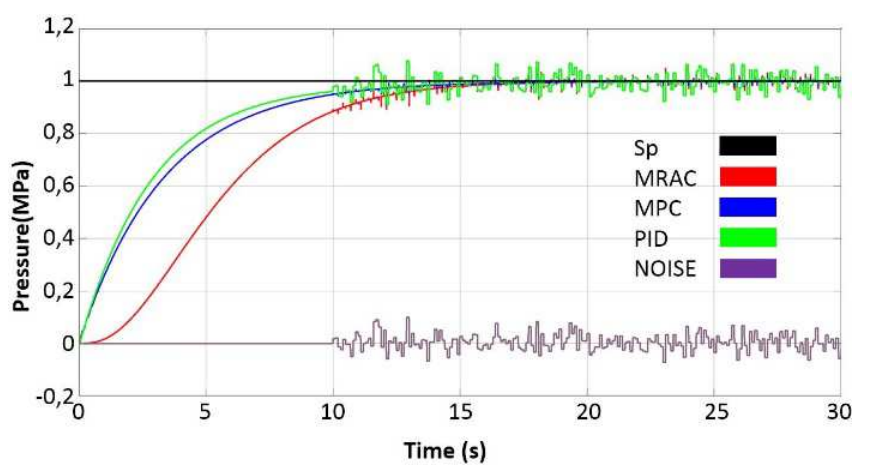

Fig. 9. Response to disturbances

From the graphs, it is found that the two systems (MPC and MRAC) respond efficiently to the variations of the reference signal and disturbances compared to the PID controller currently implemented. The MPC system responds better to external variations because the design is considering the total model of the plant. On the other hand, observing the control response, it is evident that the MRAC control has a trajectory tracking behavior. This is explained by analyzing the error signals shown in Fig. 10. 


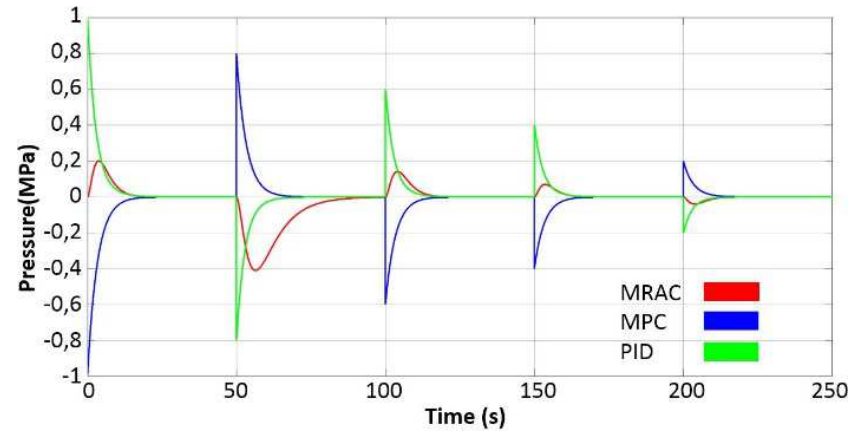

Fig. 10. Error signals

The MPC, as seen in Fig. 11 handles a constant reference like the PID, which generates over peaks in error.

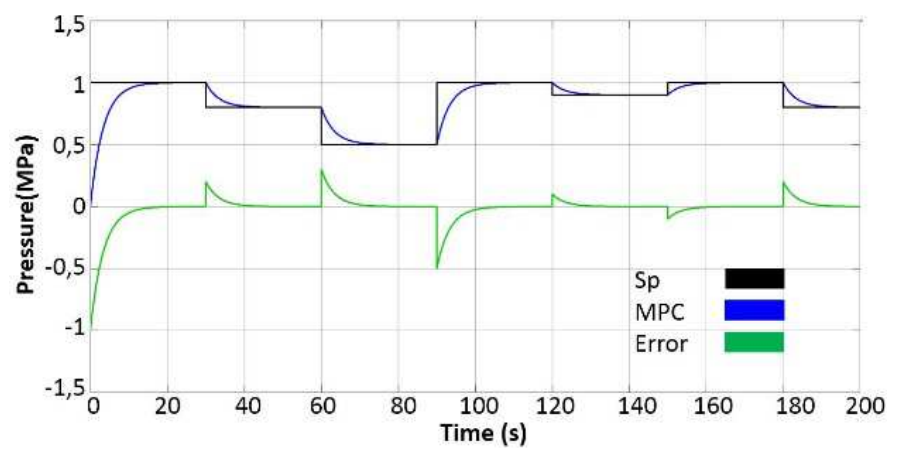

Fig. 11. MPC system

Although it should be noted that these peaks are not seen in the MPC controller response, this is because the MPC is also a reference model controller designed and predicts the transient response of the system. The MRAC controller handles a reference according to the adaptation model. The error is the difference between the models in an instant time. It suppresses the jumps and allows a tracking behavior as shown in Fig. 12.

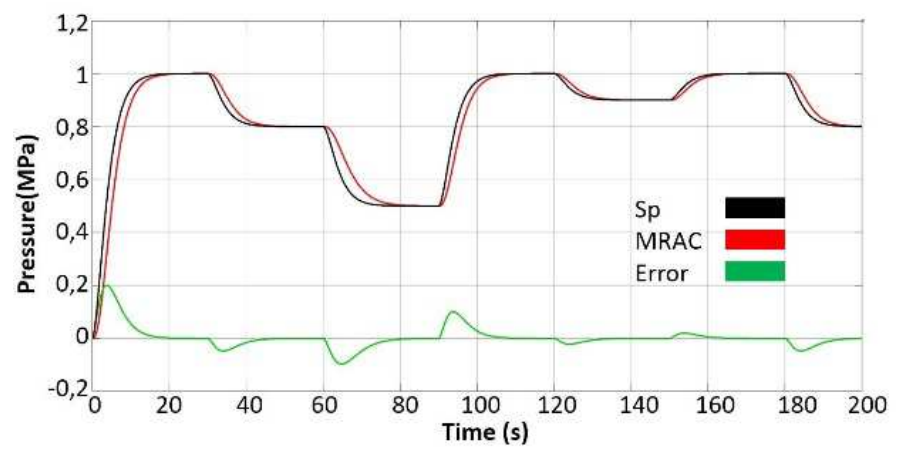

Fig. 12. MRAC system

\section{IV.CONCLUSION}

Based on the laws of Lyapunov stability and the Laguerre functions, the design of the different controllers is carried out. The results show the feasibility of its application for this type of process-the above, comparing the developed controllers with the controller currently implemented in the equipment. MPC controllers require a more extensive mathematical development in comparison to the MRAC controller and traditional controllers, but it provides a better response to disturbances.
Laguerre functions lower the computational cost in the design of the MPC controller. This is due to its recursiveness in mathematical operations and the implementation of cost and minimization functions. Also, the input parameters are lower compared to other predictive control methods. For the implementation of an MRAC, it is necessary to know the behavior of the system in an open loop. This is to design the appropriate reference model for its adaptation. One advantage of adaptive controllers is the plant stability and robustness without requiring extensive knowledge of the mathematical model from the system.

This type of controllers designed based on models reduce system power consumption by having more stable control and, in the case of MRAC, handle low error signals. The MRAC control is the one that gives the best behavior for the real equipment. Due to its smooth tracking, it simulates with greater precision a heavy load vehicle on pavements. Its implementation within a logic controller is more straightforward in industrial control environments than a MPC; this requires control equipment of greater computational capacity.

\section{ACKNOWLEDGMENT}

This product is derived from the IMP-ING-2931 highimpact project funded by the Vice-Rectory of Research at Universidad Militar Nueva Granada, to which the authors thank.

\section{REFERENCES}

[1] A. R. Pasandín and I. Pérez, "Fatigue performance of bituminous mixtures made with recycled concrete aggregates and waste tire rubber," Constr. Build. Mater. J., vol. 157, pp. 26-33, 2017.

[2] F. Moreno-Navarro, M. Sol-Sánchez, and M. Rubio-Gámez, "Structural analysis of polymer modified bituminous materials in the rehabilitation of light-medium traffic volume roads," Constr. Build. Mater., vol. 156, pp. 621-631, 2017.

[3] L. Plessis, A. Ulloa-calderon, J. T. Harvey, and N. F. Coetzee "ScienceDirect Accelerated pavement testing efforts using the Heavy Vehicle Simulator," Int. J. Pavement Res. Technol., 2018.

[4] C. Wersäll, I. Nordfelt, and S. Larsson, "Resonant roller compaction of gravel in full-scale tests," Transp. Geotech., vol. 14, pp. 93-97, 2018.

[5] J. Oravec, M. Kvasnica, M. Fikar, J. Holaza, M. Klau, and J. Drgo, "MPC-based reference governor control of a continuous stirred-tank reactor," Comput. Chem. Eng., vol. 108, pp. 289-299, 2018.

[6] M. Thiel et al., "Adaptive Model Recovery Anti-Windup for OutputFeedback plants," IFAC-PapersOnLine, vol. 1, pp. 11523-11528, 2017.

[7] D. Zhang and B. Wei, "Review article A review on model reference adaptive control of robotic manipulators," Annu. Rev. Control, vol. 43, pp. 188-198, 2017.

[8] C. Deng, G. Yang, and M. Joo, "Decentralized fault-tolerant MRAC for a class of large-scale systems with time-varying delays and actuator faults," J. Process Control, vol. 75, pp. 171-186, 2019.

[9] D. Jinjun, G. Yahui, C. Ming, and D. Xianzhong, "Adaptive variable impedance control for dynamic contact force tracking in uncertain environment," Rob. Auton. Syst., vol. 102, pp. 54-65, 2018.

[10] J. Han, S. Yu, and S. Yi, "Advanced thermal management of automotive fuel cells using a model reference adaptive control algorithm,” Int. J. Hydrog. energ y 4, vol. 2, 2016.

[11] F. D. J. Sorcia-Vázquez, C. D. Garcia-Beltran, G. Valencia-Palom, and G. Guerrero-Ramírez, "Control Predictivo Distribuido Óptimo Aplicado al Control de Nivel de un Proceso de Cuatro Tanques Acoplados,” Rev. Iberoam. Automática e Informática Ind. RIAI, vol. 12, no. 4, 2015.

[12] J. K. G. Ferramosca, D. Limon, and E. F. Camacho, "Control predictivo para seguimiento de sistemas no lineales. Aplicación a una 
planta piloto,” Rev. Iberoam. Automática e Informática Ind. RIAI, vol. 10 , no. 1,2013 .

[13] S. S. Jeremiah et al., "Generic framework for valve stiction detection and compensation with ANFIS-activated dual-mode MPC," J. Process Control, vol. 79, pp. 85-97, 2019.

[14] X. Zeng, G. Li, G. Yin, D. Song, S. Li, and N. Yang, "Model predictive control-based dynamic coordinate strategy for hydraulic hub-motor auxiliary system of a heavy commercial vehicle," Mech. Syst. Signal Process., vol. 101, no. 5988, pp. 97-120, 2018.

[15] J. K. Woodacre, R. J. Bauer, and R. Irani, "Hydraulic valve-based active-heave compensation using a model-predictive controller with non-linear valve compensations," Ocean Eng., vol. 152, no. January, pp. 47-56, 2018.

[16] A. Pai and M. Riepold, "Model-based precision position and force control of SMA actuators with a clamping application," Mechatronics, vol. 0, pp. 1-18, 2017.

[17] C. Wang, Z. Jiao, and L. Quan, "Adaptive velocity synchronization compound control of electro-hydraulic load simulator," Aerosp. Sci. Technol., vol. 42, pp. 309-321, 2015.

[18] J. Camacho-Tauta, O. Reyes-Ortiz, A. V. da Fonseca, S. Rios, N. Cruz, and C. Rodrigues, "Full-scale Evaluation in a Fatigue Track of a Base Course Treated with Geopolymers," Procedia Eng., vol. 143, pp. 18-25, 2016.

[19] W. Acuña-bravo, E. Canuto, M. Agostani, and M. Bonadei, "Proportional electro-hydraulic valves: An Embedded Model Control solution," Control Eng. Pract., vol. 62, no. January 2016, pp. 22-35, 2017.

[20] R. Ganapathy and V. Kumar, "Uniform ultimate bounded robus model reference adaptive PID control scheme for visual servoing," J. Franklin Inst., vol. 354, pp. 1741-1758, 2017.

[21] J. Han, S. Yu, and S. Yi, "Adaptive control for robust air flow management in an automotive fuel cell system," Appl. Energy, vol. 190, pp. 73-83, 2017.

[22] I. Ben Abdelwahed, A. Mbarek, and K. Bouzrara, "Adaptive MPC based on MIMO ARX-Laguerre model," ISA Trans. J., vol. 67, pp. 330-347, 2017.

[23] Y. Zheng, J. Zhou, Y. Xu, Y. Zhang, and Z. Qian, "A distributed model predictive control-based load frequency control scheme for multi-area interconnected power system using discrete-time Laguerre functions," ISA Trans. J., vol. 68, pp. 127-140, 2017.

[24] Liuping Wang, Model Predictive Control System Design and Implementation Using MATLAB, 1st ed. Springer Publishing Company, 2009. 